\title{
CORRELATION BETWEEN TPC AND TFC WITH ANTIOXIDANT ACTIVITY OF Piper sarmentosum EXTRACT AND ITS FORMULATION FOR COSMETIC PURPOSES
}

\author{
WAN ASIAH WAN SUKAIRI, LAILI CHE ROSE*, ASNUZILAWATI ASARI AND RAZIFAH \\ MOHD RAZALI
}

Faculty of Science and Marine Environment, Universiti Malaysia Terengganu, 21030 Kuala Nerus, Terengganu, Malaysia.

*Corresponding author: laili@umt.edu.my

http://doi.org/10.46754/umtjur.2021.04.005

\begin{abstract}
This study investigates the characteristics of an antioxidant cream made from the methanol extract of Piper sarmentosum leaves, which is locally known as the wild betel or pokok kadok in Malay. The secondary metabolites of the leaves were subjected to phytochemical tests to detect the presence of natural compounds. Antioxidant activity was described by its total phenolic content (TPC) and total flavonoid content (TFC), which was assessed by 2,2-Diphenyl-1-picrylhydrazyl (DPPH) assay. A phase diagram was constructed to find a possible region to formulate an antioxidant cream. In phytochemical screening, the methanolic extract showed positive presence of alkaloids, flavonoids, steroids, terpenoids and tannins. In quantitative analysis of antioxidative components, besides having significantly higher TFC content compared with quercetin $(\mathrm{P}<0.0001)$, the extract of P. sarmentosum leaves also displayed high phytochemical content and was proven to be an efficient free radical scavenger and reducing agent compared with ascorbic acid $(\mathrm{p}=0.0121)$. It was observed that the phytochemical compounds in the leaf extract like alkaloids, terpenoids, flavonoids and tannins were the major contributors of antioxidant activity. The leaf extract was also a suitable ingredient to produce a cream with good spreadability, homogeneity, consistency, appearance and $\mathrm{pH}$.
\end{abstract}

Keywords: Piper sarmentosum, antioxidant, total phenolic content, total flavonoid content, Phase diagram

\section{Introduction}

Throughout history, herbs and natural therapies have been used to improve health and they have become important ingredients in medicine and cosmetics. Some terrestrial plants, marine organisms, animals, microbes and fermentation products contain active components that may have therapeutic effects. Others have high content of anti-oxidants that have potential to enhance health and wellbeing, besides maintaining a youthful look.

Nowadays, cosmetic products are manufactured with more natural ingredients, especially those used to reduce scars. They usually come in creams, which are effective and easy to apply. Those products are a result of applying colloidal stability through microemulsion. To ensure a long shelf life, paraben is used as a preservative in cosmetic products because of its antifungal and antibacterial activity. However, there are drawbacks in using paraben in cosmetics, and many users are becoming aware of its adverse effects. Studies have found it to be carcinogenic, besides being disruptive to the endocrine system. It may also cause developmental and reproductive toxicity, and induce allergies. On the other hand, natural ingredients are more preferable as it allows the use of alternative solvents and renewable products in manufacturing, which are safer to human health (Chemat et al., 2012). Thus, this study tries to formulate a cream containing $P$. sarmentosum leaf extract using the microemulsion of non-ionic surfactants (Tween 80 and Span 65).

Piper sarmentosum or the wild betel (locally known as pokok Kadok in Malay), is a 
common herb in Southeast Asia. It mostly used for culinary purposes, but it may also be good for keeping diabetes under control. It contains different classes of antioxidative compounds like alkaloids, steroids, phenylpropanoids, C-benzylated dihydroxyflavone and phenylpropanoyl amides (Shahzad, 2017). Therefore, this study intends to explore the use of $P$. sarmentosum as a new cosmetic and skin healing product that is high in antioxidant activity.

\section{Materials and Methods}

\section{Preparation of Methanol Leaf Extract}

\section{Alkaloid detection}

Mayer's test was used to detect the presence of alkaloids, with a small amount of leaf extract added to diluted $\mathrm{HCl}$ and mixed with Mayer's reagent. Presence of alkaloids was determined with the formation of yellow precipitates (Tiwari et al., 2011).

\section{Flavonoid detection}

The alkaline reagent test was used to detect flavonoids. Two to three drops of $\mathrm{NaOH}$ solution were added into the leaf extract as described by Tiwari et al. (2011). The presence of flavonoids was confirmed when the solution turned intense yellow and become colorless again with the addition of a diluted acid.

\section{Steroid detection}

The Liebermann-Burchard test was used to detect steroids using the protocols described by Singh et al. (2012). Briefly, $0.5 \mathrm{ml}$ of the extract was mixed with $2.0 \mathrm{ml}$ of acetic anhydride. The mixture was then cooled in a bowl of ice. About $1.0 \mathrm{ml}$ of concentrated $\mathrm{H}_{2} \mathrm{SO}_{4}$ was added and the appearance of a blue green ring would confirm the positive presence of steroids (Singh et al., 2012).

\section{Detection of Terpenoids}

Terpenoids were detected using the Salkowski Test. A total of $5.0 \mathrm{ml}$ extract was mixed with $2.0 \mathrm{ml}$ chloroform. Concentrated $\mathrm{H}_{2} \mathrm{SO}_{4}$ was added carefully to form a single layer in the organic solution. Formation of a reddish-brown layer at the interface would indicate the presence of terpenoids (Mir \& Sawhney, 2013).

\section{Detection of Tannins}

Tannins were detected using the bromine water test. A total of $1 \mathrm{ml}$ extract was added with equal amount of bromine water. A reddish-brown precipitate or greenish black formation would prove the presence of tannins in the leaf extract (Singh et al., 2012).

\section{Determination of Total Phenolic Content}

TPC was determined using the Folin-Ciocalteu reagent as described by Velioglu et al. (1998). The methanolic leaf extract was diluted three times with methanol and mixed with $2.25 \mathrm{ml}$ of Folin-Ciocalteu reagent in triplicates before being left in the dark at room temperature for five minutes. A total of $2.25 \mathrm{ml}$ of $60 \mathrm{~g} / \mathrm{L}$ sodium carbonate solution was added and the mixtures were allowed to sit in room temperature for another 90 minutes before the absorbance was measured using a spectrophotometer at $725 \mathrm{~nm}$ wavelength. Gallic acid was used as a standard to compare with the extract.

\section{Determination of Total Flavonoid Content}

TFC was studied using the calorimetric method according to Dewanto et al. (2002). A total of $0.5 \mathrm{ml}$ of methanolic extract was dissolved with $2.25 \mathrm{ml}$ distilled water in triplicate test tubes. Then, $5 \%$ NaNO2 $(0.15 \mathrm{ml})$ solution was added to the mixtures. Approximately after six minutes, $0.3 \mathrm{ml}$ of $10 \% \mathrm{AlCl}_{3} .6 \mathrm{H} 2 \mathrm{O}$ solution was added and the solutions were left in room temperature for five more minutes. Then, an aliquot of $1 \mathrm{M}$ $\mathrm{NaOH}(1.0 \mathrm{ml})$ was added. The absorbance was measured at a wavelength of $510 \mathrm{~nm}$. Quarcetin was used to compare flavonoid content with the leaf extract. 


\section{DPPH Free Radical Scavenging Assay}

The free radical scavenging activity of the leaf extract was measured with 1,1-diphenyl-2picrylhydrazyl (DPPH) scavenging assay (Magalhaes et al., 2006). An aliquot of samples with various concentration diluted in $80 \%$ methanol were prepared in triplicates with $3.0 \mathrm{ml}$ of $0.5 \mathrm{M}$ DPPH in absolute ethanol, which was then allowed to stand at ambient temperature for 30 minutes in the dark. The mixtures' absorbance was then measured using a spectrophotometer at a wavelength of $520 \mathrm{~nm}$. The percentage of free radical scavenging was calculated using Equation 1 (Eq 1).

Free radical scavenging activity $(\%)=\frac{\text { Ac-As }}{A c} \times 100$

whereas is the absorbance of the sample and Ac is absorbance of negative control at $520 \mathrm{~nm}$ wavelength.

\section{Statistical analysis}

All results were presented in mean \pm standard error (SEM). The IBM SPSS version 20.0 software (IBM Corp, Armonk, NY, USA) was used to analyse the data and statistical tests performed included one-way ANOVA $(\mathrm{P}<0.05)$, unpaired $t$ test $(p<0.05)$ and Pearson correlation test.

\section{Construction of phase diagram}

The titration method was used to construct the ternary phase diagram. A total of $0.5 \mathrm{~g}$ Tween 80 and Span 65 mixture was dissolved in nine vials of constant heptane at ratios of 1:9, 2:8, $3: 7,4: 6,5: 5,6: 4,7: 3,8: 2$ and 9:1, respectively. A very small amount of deionized water was titrated into each vial and the mixtures were vortexed until they became homogenised with each titration of water. This was followed by centrifugation at $3500 \mathrm{rpm}$ for 10 minutes. The samples were observed for phase separation or changes.
For the phase diagram, the solutions that were observed as clear and isotropic were classified as one phase micro-emulsion, while solutions that were cloudy in one phase were classified as one phase macro-emulsion. The process was repeated with the addition of butanol to broaden the phase region.

\section{Cream Formulation}

All ingredients were measured according to reference formulation in Table 1. Oil phase and aqueous phase were separated in beakers and heated in a water bath at $70^{\circ} \mathrm{C}$ for $10-15$ minutes. The oil phase was poured into the aqueous phase and stirred thoroughly until an emulsion was formed. A homogenizer was used to homogenize the emulsion into a fine, smooth texture. The cream was allowed to cool down before being divided in two air-tight vials. The $P$. sarmentosum leaf extract was added into one vial and stirred until it mixed evenly while the other vial without the leaf extract was used as a comparison control.

Table 1: Modified formulation and ingredients of cream emulsion

\begin{tabular}{ccc}
\hline & $\mathbf{\%}$ & Weight $\mathbf{( g )}$ \\
\hline Cetyl alcohol & 10.00 & 3.00 \\
Olive oil & 20.24 & 6.07 \\
Tween 80 & 23.76 & 7.13 \\
$\mathrm{H}_{2} \mathrm{O}$ & 43.00 & 12.90 \\
Glycerine & 1.00 & 0.33 \\
P. sarmentosum leaf & 2.00 & 0.60 \\
extract & & \\
\hline Total & 100.00 & 30.00 \\
\hline
\end{tabular}

\section{Physical properties of cream and evaluation}

The two prepared formulation with and without plant extract were examined and evaluated for their physical properties which is based on their texture, homogeneity and $\mathrm{pH}$.

Organoleptic characteristics: Each formulation was tested for color texture, physical appearance and homogeneity. These characteristics were evaluated by visual 
observation. Homogeneity and texture were tested by pressing a sufficient amount of each formulated cream sample between the thumb and index finger. The consistency of the cream and presence of coarse particles in it were used to determine the texture and homogeneity of the cream sample. Immediate feels on the skin such as stiffness, grittiness, and greasiness were also tested.

Determination of $\mathbf{p H}$ : $1 \mathrm{~g}$ from each of the cream sample was measured and mixed in $25 \mathrm{ml}$ of deionized water to obtain liquid form in order to fill it in the digital $\mathrm{pH}$ meter. The digital the $\mathrm{pH}$ meter used is Laquatwin $\mathrm{pH}$ meter, USA. Before conducting the measurements, the $\mathrm{pH}$ meter was calibrated with $\mathrm{pH} 7$ buffer solution followed by $\mathrm{pH} 4$ first. The measurements were made 3 times to calculate the mean.

Spreadability (S): A sufficient amount of cream sample from each formulation were applied and compressed to uniform thickness between two glass slides. The glass slides then were loaded with $100 \mathrm{~g}$ weight for 7 minutes and was observed. The time taken for the two glass slides to separate was recorder to measure the stability. The less the time taken for the glass slide to separate, the greater the spread ability

of the cream. The spread ability value was then calculated using Equation 2 (Eq 2).

$$
\mathrm{S}=(\mathrm{m} \cdot 1) / \mathrm{t}
$$

where $\mathrm{S}$ is the spreadability, $\mathrm{m}$ is the weight applied on the upper glass slide per gram, 1 is the length of the upper glass slide moved on the lower plates of the glass slide per $\mathrm{cm}$, and $\mathrm{t}$ is the time taken for the glass slide to separate per second.

The spreadability test was also conducted by applying the cream samples directly onto the skin at the back of the palm and observed whether the spread was even and good.

\section{Results and Discussion}

The plant sample contained all five secondary metabolites which was beneficial in treatment of wounds. Some of them were advantageous and important as antioxidants, which would promote wound healing. For example, flavonoids are major antioxidants that fight free radicals that could damage the skin. The results of the phytochemical screening are tabulated in Table 2 .

Table 2: Summary of phytochemical screening results $P$. sarmentosum extract

\begin{tabular}{cccc}
\hline No. & Natural compound test & Result & Conclusion \\
\hline 1 & Alkaloids & Formation of yellow color precipitate & Presence of alkaloids \\
2 & Flavonoids & Intense yellow color to colorless & Flavonoids present \\
3 & Steroids & Formation of blue- green ring & Presence of steroids \\
4 & Terpenoids & Reddish-brown coloration interface & Terpenoids present \\
5 & Tannins & Reddish-brown precipitate & Presence of tannins \\
\hline
\end{tabular}

\section{Total Phenolic Content}

Phenolic compounds are all popular in many plants and herbs as they act as antioxidant and free radical scavengers. A study is carried out to determine TPC in the leaves of Piper sarmentosum. The plant extract was compared with standard gallic acid in this study to quantify the compound present in sample, and the light absorbance was measured using a UV-Vis spectrophotometer with wavelength of $725 \mathrm{~nm}$. The values for TPC are shown in Figure 1. From the figure, it is obvious that TPC in gallic acid was higher than in P. sarmentosum.

For this study, unpaired test was used to compare the TPC between P. sarmentosum and standard gallic acid. The study showed that there was a statistically high significant difference $(p<0.01)$ in TPC between P. sarmentosum and 
gallic acid (Figure 1), which showed a mean \pm Standard Error Mean (SEM) of $0.4209 \pm$
0.1105 and $1.000 \pm 0.0$, respectively. The $95 \%$ confidence interval was 0.2724 to 0.8858 .

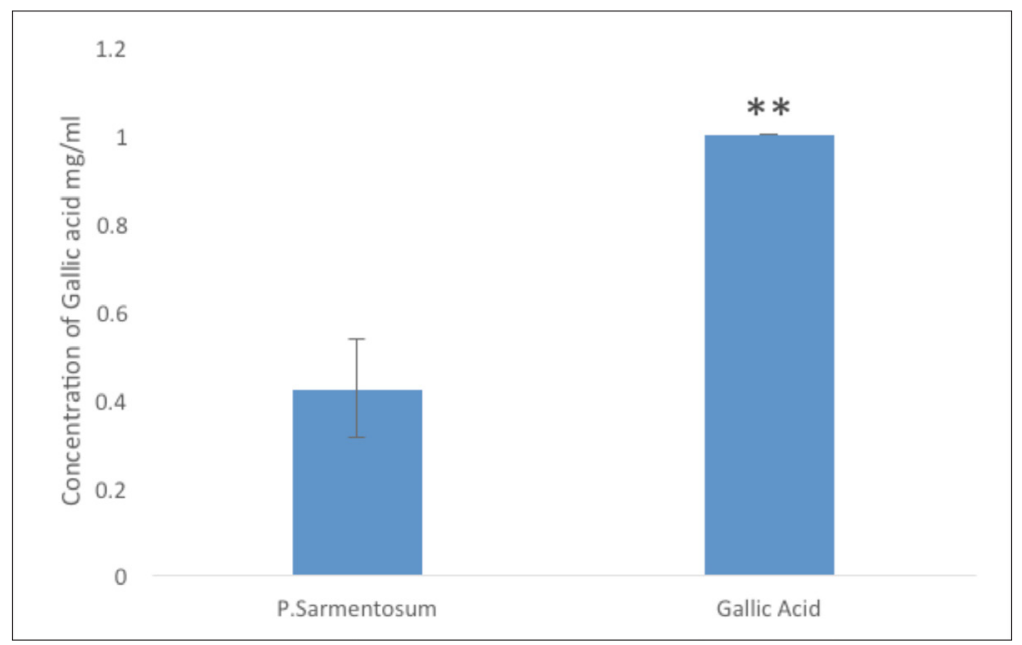

Figure 1: Total phenolic content of Piper sarmentosum and gallic acid. ** indicates significant difference $(p<0.01)$. Error bars represent standard deviation

\section{Total Flavonoid Content}

The study shows that the Total Flavonoid Content (TFC) found was determined in comparison with standard quercetin. It was proven that the TFC content in P. sarmentosum was significantly higher than quercetin. The plant extract was compared with standard gallic acid to quantify the compound present in the sample. The values for TFC are shown in Figure
2. From the figure, it was obvious that TFC in $P$. sarmentosum was higher than in quercetin. For this study, unpaired t test was used to compare the TFC between $P$. sarmentosum and quercetin, and a very significant difference $(p<0.0001)$ was observed0. The mean concentration of TFC in the leaf extract and quercetin was $36.75 \pm$ 0.1146 and $1.000 \pm 0.0$, respectively. The $95 \%$ confidence interval was 36.07 to -35.44 .

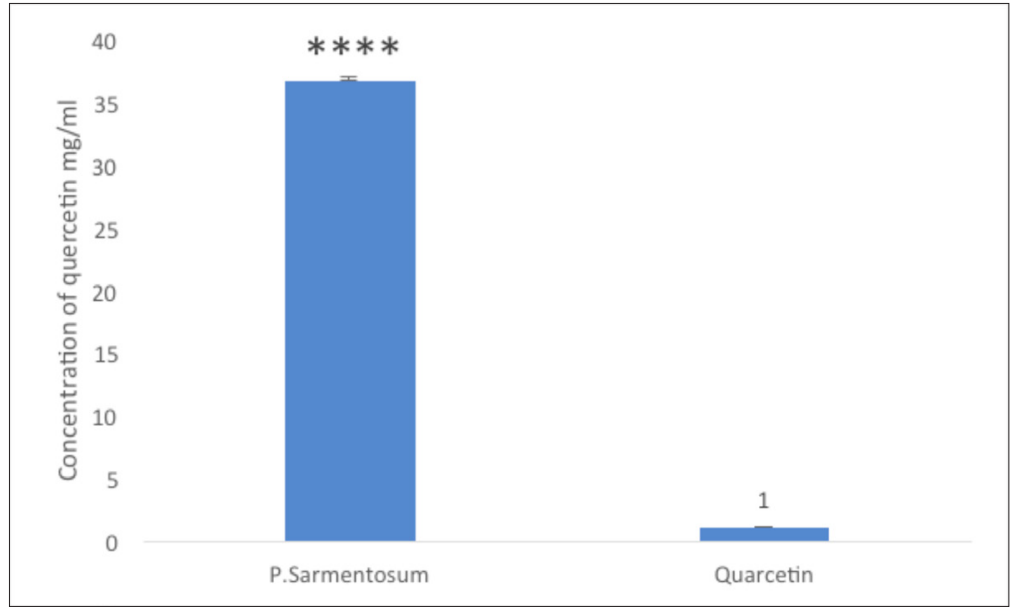

Figure 2: Total flavonoid content of Piper sarmentosum and quercetin. ****indicates significant difference $(p<0.0001)$. Error bars represent standard deviation 


\section{DPPH Scavenging Assay}

The antioxidant activity of the leaf extract was determined through DPPH (2,2-diphenyl-1picrylhydrazyl) scavenging assay with ascorbic acid as a positive control. A negative control was also performed to calculate the percentage of scavenging effect. The leaf extract displayed excellent scavenging effect on DPPH radicals, which was in the range of above 1,500\% compared to ascorbic acid, which was below the range of $250 \%$ at concentration of $3 \mathrm{mg} / \mathrm{ml}$ (Figure 3). Unpaired t test showed a significant difference between the antioxidant activity of $P$. sarmentosum extract and ascorbic acid $(\mathrm{P}<0.05)$, which had a mean scavenging percentage of $1,518 \pm 296.8$ and $226.2 \pm 0.4496$, respectively. The $95 \%$ confidence interval was -2116 to -468.0. The $\mathrm{F}$ test also found a significant difference between the variances $(p<0.0001)$.

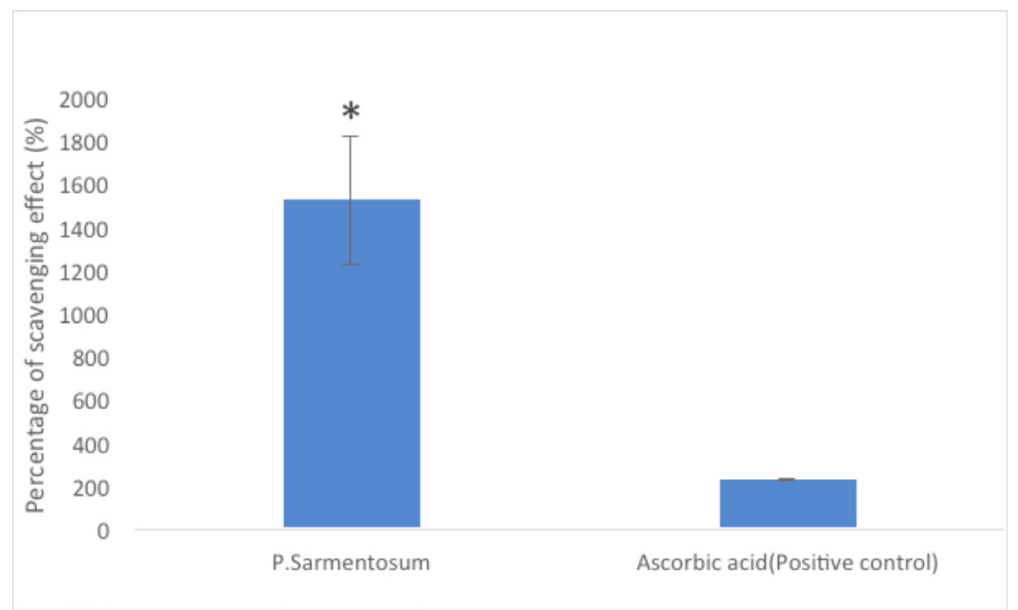

Figure 3: The scavenging activity of Piper sarmentosum and ascorbic acid assayed by DPPH free-radical scavenging method. * indicates significant difference $(p<0.05)$. Error bars represent standard deviation

The antioxidant in the sample would turn from purple to yellow as it was oxidized by DPPH. The percentage of scavenging effect was very high probably because the total antioxidant content in the sample was lower than those in the absorbance control. Apart from that, the DPPH coloration might also be too concentrated or had been slightly damaged due to light exposure.

\section{Correlation}

An analysis of correlation was used to study phytochemical contents and antioxidant activity of P. sarmentosum. Correlations between TPC, TFC and antioxidant activities were analyzed using Pearson correlation as stated in Table 3. From the table, the DPPH assay had positive correlation with TPC and TFC ( $\mathrm{r}=0.9343$ and $\mathrm{r}=1$, respectively). It was proven that phytochemicals such as TPC and TFC present in $P$. sarmentosum basically contributed to the total antioxidant activity that it exhibited. Both showed a correlation in the assay, therefore, TPC and TFC might be major contributors of the antioxidant activity in the plant extract.

Table 3: Pearson correlation analysis (r values) of TPC, TFC and antioxidant activity

\begin{tabular}{cc}
\hline TPC & $0.9343 * *$ \\
TFC & $1 * * * *$ \\
\hline
\end{tabular}

$* *$ Correlations were significant $(\mathrm{p}<0.01)$

$* * * *$ Correlations were significant $(\mathrm{p}<0.0001)$

\section{Construction of Phase Diagrams}

A ternary phase diagram was constructed to find the suitable region for formulating a cream 
containing a natural ingredient. In order to do so, a ternary phase diagram consisting of water and non-ionic emulsifiers was constructed. Along the process, the phase behavior of the system was observed and the results were presented in the diagram. An oil phase used in this study was heptane, whereas the non-ionic surfactants were Tween 80 (Polyoxyethylene 20 Sorbitan Monooleate) and Span 65 (Sorbitan Tristearate), which is shown in Figure 4.

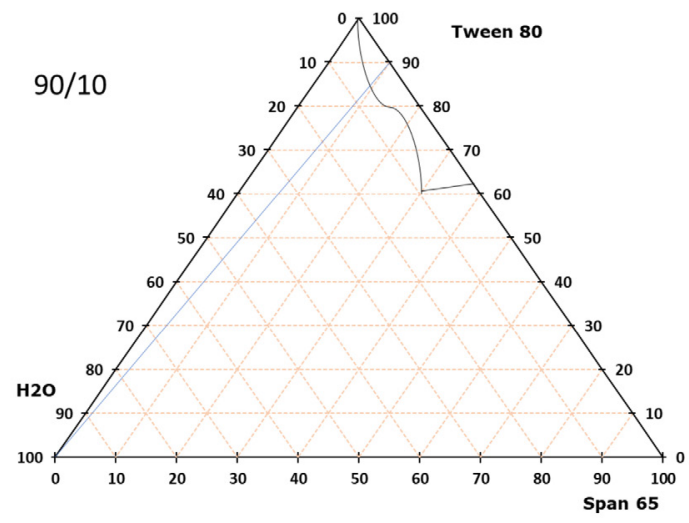

Figure 4: Ternary phase diagram of Water-Tween 80-Span 65 system with heptane as an oil phase

Tween $80(\mathrm{HLB}=15)$ and Span 65 (HLB $=2.1$ ) were chosen since they were readily available in the laboratory and their colloidal structures had been characterized by many groups as well as other researches. Figure 4 shows the area of the one-phase isotropic region for water/Span65/Tween 80 with heptane $50 \%$ as a constant oil phase. It was observed that both
Tween 80 and Span 65 were completely miscible with each other. A maximum water solubility of seven weight per cent of water was observed at Tween 80 to Span 65 weight ratio of 90:10. The isotropic solution region was observed to be very small on the ternary phase diagram. So, another phase diagram with the addition of butanol (Figure 5) was drawn to obtain a larger isotropic region.

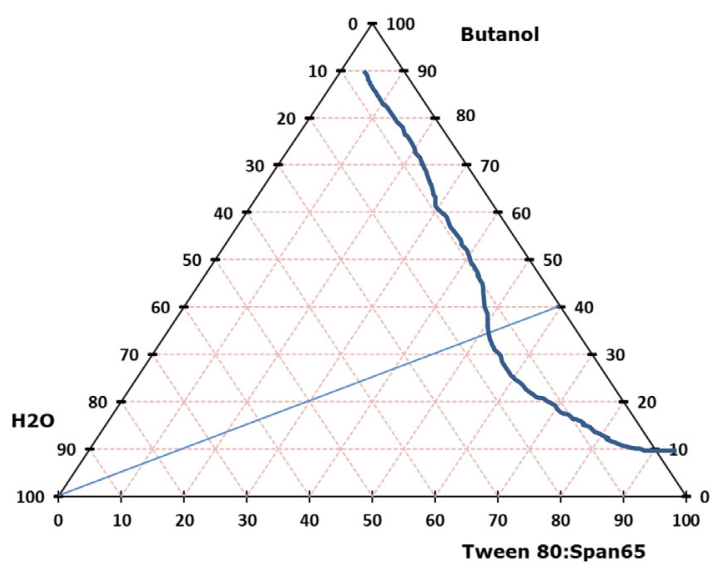

Figure 5: Ternary phase diagram of Water-Butanol-Tween 80 to Span 65 system with heptane as an oil phase 
The 90:10 ratio (Tween 80 to Span 65) was then used as the mixed non-ionic surfactant apex and titrated with a third component, which was butanol. The figure showed a similar pattern of the isotropic region to that of the water/Tween 80/Span 65 system, but with an increased amount of the maximum water solubility of 20 $\%$ by weight. The maximum water solubility occurred at the butanol/Tween 80 to Span 65 (90:10) weight ratio of 40:60. This suggested that a higher amount of mixed surfactants were required to achieve the maximum water solubility and to form the colloidal structures. However, the region was still not suitable to formulate a cream due to its unstable phase and narrow region. Thus, a formulation derived from a ternary phase diagram constructed by Doreen (2008) with slight modification of essential oils was adapted to formulate two formulations of cream samples for this study.

\section{Cream Formulation and Characterization}

Organoleptic characteristics

Table 4 shows the results of the organoleptic characteristics for both cream samples with and without $P$. sarmentosum leaf extract. From the results, the mean $\mathrm{pH}$ of the cream with the extract was 4.28 and 4.70 for the cream without (Table 5). The $\mathrm{pH}$ of the creams was suitable to apply on skin as the $\mathrm{pH}$ of human skin was in the range of 4 to 6 .

Table 4: The organoleptic characteristics of cream samples with and without $P$. sarmentosum leaf extract

\begin{tabular}{cccc}
\hline No. & Organoleptic properties & With plant extract & Without plant extract \\
\hline 1 & Physical appearance & Opaque & Opaque \\
2 & Colour & Green & White \\
3 & Texture & Viscous & Viscous \\
4 & Homogeneity & Present & Present \\
5 & Immediate skin feeling & Greasiness, moisturizing, & Greasiness, moisturizing, \\
& & spreadable & spreadable \\
\hline
\end{tabular}

Table 5: The $\mathrm{pH}$ readings in both of cream samples

\begin{tabular}{cccc}
\hline No. & Tests & With plant extract & Without plant extract \\
\hline 1 & First test & 4.42 & 4.89 \\
2 & Second test & 4.19 & 4.79 \\
3 & Third test & 4.24 & 4.42 \\
$\mathbf{4}$ & Mean & $\mathbf{4 . 2 8}$ & $\mathbf{4 . 7 0}$ \\
\hline
\end{tabular}

\section{Spreadability}

The spreadability of both cream samples were calculated using Equation 2. Spreadability is an ability of a cream or gel to be evenly spread on the skin. From the calculation, the spreadability mean of cream sample with and without plant extract was $26.94 \mathrm{~g} \mathrm{~cm} / \mathrm{s}$ and $25.85 \mathrm{~g} \mathrm{~cm} / \mathrm{s}$, respectively, as shown in Tables 6 and 7. The result of spreadability denoted the extent of area to which the prepared formulations readily spread on application to skin, while homogeneity ensured that no lumps were formed (Pattanayak et al., 2011).

Table 6: Spreadability of cream sample with plants extract

\begin{tabular}{cc}
\hline First test & $S=\frac{100.2 .5}{12}=20.83 \mathrm{gcm} / \mathrm{s}$ \\
Second test & $S=\frac{100.3 .5}{10}=35.00 \mathrm{~g} \mathrm{~cm} / \mathrm{s}$ \\
Third test & $S=\frac{100.3 .0}{12}=25.00 \mathrm{~g} \mathrm{~cm} / \mathrm{s}$ \\
Mean & $26.94 \mathrm{~g} \mathrm{~cm} / \mathrm{s}$ \\
\hline
\end{tabular}


Table 7: Spread ability of cream sample without plants extract

\begin{tabular}{cc}
\hline First test & $S=\frac{100.2 .5}{13}=19.23 \mathrm{~g} \mathrm{~cm} / \mathrm{s}$ \\
Second test & $S=\frac{100.3 .0}{12}=25.00 \mathrm{~g} \mathrm{~cm} / \mathrm{s}$ \\
Third test & $S=\frac{100.3 .0}{9}=33.33 \mathrm{~g} \mathrm{~cm} / \mathrm{s}$ \\
Mean & $25.85 \mathrm{~g} \mathrm{~cm} / \mathrm{s}$ \\
\hline
\end{tabular}

The sample creams were tested directly at the back of the palm. When it was applied and rubbed on the skin, the creams exhibited decreasing viscosity. The more the creams were rubbed onto the skin, the easier they spread.

\section{Conclusion}

There is a strong positive correlation between TPC and TFC with free radical scavenging activity of the P. Sarmentosum leaf extract, thereby showing it was a good source of antioxidants and may have the potential to become a product that can promote wound healing. A previous study had found that the plant extract was biocompatible and non-toxic towards human liver tissue. Plus, the plant extract had also been studied for its potential in reducing blood sugar levels. The medicinal potential of $P$. Sarmensotum should be explored further, especially in experiments to see how efficient it promotes wound healing and prevent the formation of scars.

\section{Acknowledgments}

The authors are thankful to the university for the research opportunity and the reviewers for the constructive comments.

\section{References}

Ab Rahman, S. F. S., Sijam, K., \& Omar, D. (2014). Chemical composition of Piper sarmentosum extracts and antibacterial activity against the plant pathogenic bacteria Pseudomonas fuscovaginae and Xanthomonas oryzae pv. oryzae. Journal of Plant Diseases and Protection, 121(6), 237-242.

Aslam, M. S., Ahmad, M. S., Ahmad, M. A., \& Akhlaq, M. (2017). An updated review on phytochemical and pharmacological propeties of piper sarmentosum. Current Trends in Biotechnology and Pharmacy, 11(4), 345-356.

Chanprapai, P., \& Chavasiri, W. (2017). Antimicrobial activity from piper sarmentosum roxb. against rice pathogenic bacteria and fungi. Journal of integrative agriculture, 16(11), 2513-2524.

Dumbarwadi, P. (2011). Cajanus scarabaeoides. Journal of Pharmacy and Allied Health Sciences, 1(2), 49-57.

Hamil, M. S. R. (2016). Standardization, safety and anti-obesity studies of piper sarmentosum roxburgh extracts (Doctoral dissertation, Universiti Sains Malaysia).

Kasemweerasan, P. (2015). Antioxidant activity of piper sarmentosum roxb. and its effect on the degradation of frying oils (Doctoral dissertation, University of Leeds).

Laili, C. R., Doreen, N. S., \& Hamdan, S. (2018). Optical pattern of liquid crystalline structure in singular and mixed anioniccationic surfactant system. Oriental Journal of Chemistry, 34(1), 110-114.

Laili, C. R., \& Hamdan, S. (2015). Phase Diagrams of W/O microemulsion stabilised by nonionic surfactants to be used as templates for microemulsion polymerisation. Oriental Journal of Chemistry, 31(3), 1595-1599.

Li, Y., Fabiano-Tixier, A. S., Vian, M. A., \& Chemat, F. (2013). Solvent-free microwave extraction of bioactive compounds provides a tool for green analytical chemistry.

TrAC Trends in Analytical Chemistry, 47, $1-11$.

Medini, F., Fellah, H., Ksouri, R., \& Abdelly, C. (2014). Total phenolic, flavonoid and tannin contents and antioxidant and antimicrobial activities of organic extracts of shoots of 
the plant Limonium delicatulum. Journal of Taibah University for science, 8(3), 216224.

Mohd Zainudin, M., Zakaria, Z., Nordin, M. M., Anita, N., \& Othman, F. (2013). Does oral ingestion of Piper sarmentosum cause toxicity in experimental animals? Evidence Based Complementary and Alternative Medicine, 2013.

Moldovan, M., Lahmar, A., Bogdan, C., Părăuan, S., Tomuta, I. \& C, M. (2017). Formulation and evaluation of a water-in-oil cream containing herbal active ingredients and ferulic acid. Clujul Medical, 90(2), 212.

Narayanaswamy, R., \& Ismail, I. S. (2015). Cosmetic potential of Southeast Asian herbs: an overview. Phytochemistry Reviews, 14(3), 419-428.

Pyrzynska, K., \& Pękal, A. (2013). Application of free radical diphenylpicrylhydrazyl (DPPH) to estimate the antioxidant capacity of food samples. Analytical Methods, 5(17), 4288-4295.
Singh R., Kumar A., Giri D. D., B. K., \& P. K. D. (2012). Gas chromatogrphy- mass spectrometry analysis and phytochemical screening of methanolic fruit extract of momordica charantia. Journal of Recent Advances in Agriculture, 1(4), 122-127.

Velioglu, Y. S., Mazza, G., Gao, L., \& Oomah, B. D. (1998). Antioxidant activity and total phenolics in selected fruits, vegetables, and grain products. Journal of agricultural and food chemistry, 46(10), 4113-4117.

Wang, W., Wang, Y. J., \& Wang, D. Q. (2008). Dual effects of Tween 80 on protein stability. International journal of pharmaceutics, 347(1-2), 31-38.

Yoshida, M., Suzuki, M., Susa, K., \& Kurumada, K. (2005). The effect of surfactants on liquid liquid extraction of ceria nanoparticles. Journal of chemical engineering of Japan, 38(9), 718-721.

Zainudin, M. M., Zakaria, Z., \& Nordin, N. A. M. M. (2015). The use of Piper sarmentosum leaves aqueous extract (Kadukmy ${ }^{\mathrm{TM}}$ ) as antihypertensive agent inspontaneous hypertensive rats. BMC complementary and alternative medicine, 15(1). 